\title{
More than 100 countries still not using global outbreak surveillance regulations
}

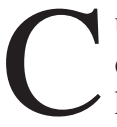

urrent outbreaks of a novel coronavirus and avian influenza have come to world attention quickly, thanks to a revamped global system of disease surveillance and a vastly expanded role for the World Health Organization (WHO) under the International Health Regulations (IHR). However, the regulations guiding this surveillance, which were adopted postSARS in 2005, are not yet applied in 110 of the 195 WHO-member countries.

These regulations, which call on member states to develop national surveillance and response systems, "represent a potentially revolutionary change in global health governance," stated Dr. Kumanan Wilson, a public health researcher with the Ottawa Health Research Institute, University of Ottawa in Ontario in a recent article (Health Policy Plan 2010;25:505-9).

"It's critical, and it's one of the most important things that has happened in the world of outbreaks," agrees Dr. Isabelle Nuttall, director of the Global Capacities, Alert and Response department of WHO.

She says an early benefit of the regulations is "a much more open dialogue between WHO and the member states."

On its website, WHO acknowledges active cooperation from public health authorities in Saudi Arabia and China during the current outbreaks. A Canadian source working in China confirms that China's handling of the avian influenza outbreak is "a major advance over previous policies." Delays in confirming the original SARS outbreak in
China were a factor in the international push for stronger regulations.

"The worst-case scenario is that we could have detected something at source but failed to," explains Wilson. "That's why IHR was brought in, and SARS was why it was brought in, really."

Yet bringing all WHO-member countries to high standards for compliance has proved difficult. While the regulations took effect in 2007, countries had until 2012 to comply. Canada attained full compliance by the deadline, but Nuttall says that 110 countries - $56 \%$ of the 195 member states have asked for a two-year extension allowed under the regulations. Nuttall says that even some industrialized countries such as France and the United Kingdom have asked for extensions,

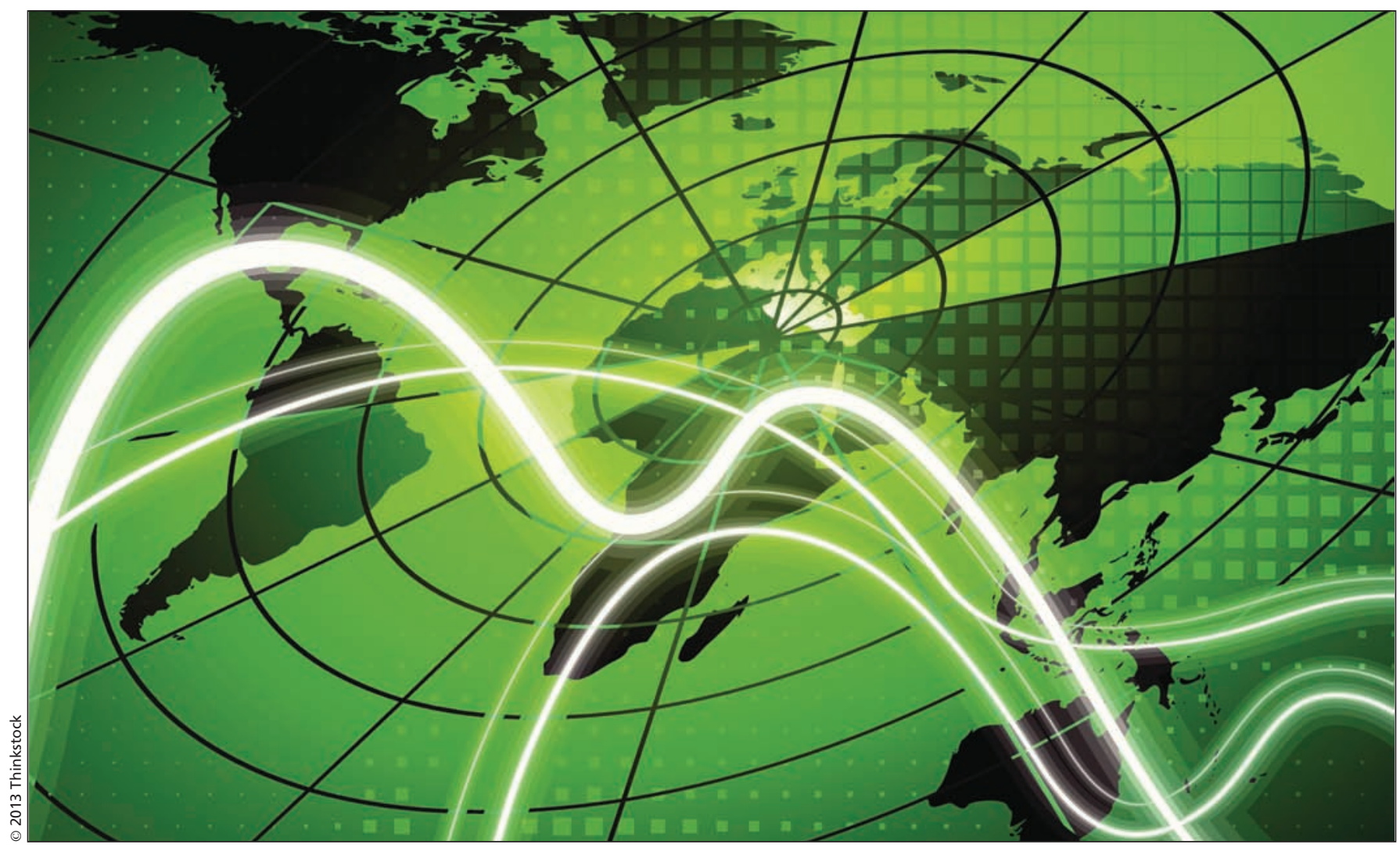

National surveillance and response systems are a revolutionary change in global health governance. 
but many developing countries are much farther from compliance.

"Some of these countries are dealing with their own epidemics of malaria and HIV," Wilson points out. "There's not a whole lot of incentive for them to take their limited resources away from the issues they're facing to detect a disease."

Nuttall emphasizes that WHO provides countries with assistance such as guidelines, standards, training and help from about 300 institutions in member countries, but it does not provide direct funding. "We're working hard to support them, but whenever there is no big crisis there is a lack of attention paid."

Wilson says the H1N1 influenza epidemic in 2009 highlighted that a dearth of high-quality surveillance in Mexico contributed to delays in identifying the outbreak and mistakes in estimating its severity. Since surveillance is key to the WHO pandemic influenza strategy, it can affect response during outbreaks such as the current ones.

Before 2005, member states were required to report only a very limited list of known diseases (cholera, plague and yellow fever) to WHO. When an outbreak occurred, there was a lack of communication channels between WHO and national public health authorities.

"During the SARS outbreak, we had no good system in place. We had to use the media" to inform member countries, says Nuttall.

Under IHR, member states must develop national surveillance and response systems, including public health capacity at designated ports, airports and ground crossings. Countries must also designate a "national focal point" for immediate communication with the WHO using a secure website.

As well, member countries are obligated to report all types of public health emergencies that meet any two of four straightforward criteria: serious public health impact, unusual or unexpected nature, risk of international disease spread and risk that travel or trade restrictions will be imposed by other countries.
Furthermore, WHO can now investigate what Nuttall calls "rumours" about a public health problem.

"We have a system in place that is constantly screening the media and the Web," she explains, which can trigger WHO to conduct its own assessment. While most media and Internet reports turn out to be unfounded, if WHO is concerned about a potential public health problem, it can require a state to provide verification within 48 hours.

Since countries have started putting IHR measures in place, Nuttall says there is marked improvement. "What we are seeing is that cases are being reported to us. We are able to inform much more rapidly, national authorities are able to take action much more rapidly, and the international threat of disease is decreased. Most countries are much more reactive than 10 years ago, that's for sure." - Carolyn Brown, Ottawa, Ont.

CMAJ 2013. DOI:10.1503/cmaj.109-4479 